\title{
Energy Efficient Design for OFDM-Based Underlay Cognitive Radio Networks
}

\author{
Yunli Chen, ${ }^{1}$ Zhengguang Zheng, ${ }^{2}$ Yibin Hou, ${ }^{1}$ and Yong $\mathrm{Li}^{1}$ \\ ${ }^{1}$ Institute of Embedded Software and Systems, Beijing University of Technology, Beijing 100022, China \\ ${ }^{2}$ Department of Vehicular Information Systems, North Information Control Group Co., Ltd., Nanjing 211153, China
}

Correspondence should be addressed to Zhengguang Zheng; zhguangzheng@gmail.com

Received 9 January 2014; Accepted 3 April 2014; Published 22 April 2014

Academic Editor: Trung Nguyen Thoi

Copyright (C) 2014 Yunli Chen et al. This is an open access article distributed under the Creative Commons Attribution License, which permits unrestricted use, distribution, and reproduction in any medium, provided the original work is properly cited.

Conventional designs on OFDM-based underlay cognitive radio (CR) networks mainly focus on interference avoidance and spectral efficiency (SE) improvement. As green radio becomes increasingly important, this paper investigates energy efficient power allocation. Our aim is to maximize energy efficiency (EE), subject to the constraints on the total transmit power, the peak interference power, and the minimum data rate requirement. We first analyze the relationship between SE and EE and solve this optimization problem with the help of bisection search technique. However, the accuracy of the power allocation solution is dependent on the number of iterations. In order to achieve the exact optimal solution, a new energy efficient power allocation scheme is proposed to balance the tradeoff between SE and EE. Simulation results are provided to demonstrate the effectiveness of the proposed schemes.

\section{Introduction}

The rapid development of wireless communication applications has given rise to a tremendous demand for scarce spectrum resources and energy conservation [1]. Since a large number of licensed frequency bands are underutilized, cognitive radio (CR) has been viewed as a promising technology to improve spectral efficiency (SE) via spectrum sharing [2].

Orthogonal frequency division multiplexing (OFDM), which has been regarded as a leading technique for high-data rate transmission due to its inherent resistance to multipath fading and the flexibility in resource allocation, has received wide attention in the evolution and development of CR technology [3]. In order to protect primary user (PU) activity, the interference caused by secondary user (SU) transmitter $(\mathrm{Tx})$ at $\mathrm{PU}$ receiver $(\mathrm{Rx})$ should be maintained below a target threshold [4].

Power allocation is a critical design issue for CR networks. Conventional power allocation for OFDM-based CR networks mainly focused on SE [5-7], which measures how efficiently a spectrum band is utilized. However, maximizing the SE may not lead to a high energy efficiency (EE), which is defined as the number of transmitted bits per unit energy [8].
To this end, power allocation schemes to maximize EE have been studied. In [9], the authors discussed joint transmission duration and power allocation to achieve the maximum EE. The authors in [10] investigated energy efficient power allocation schemes in heterogeneous CR networks with femtocells. However, the quality of service (QoS) of SU is ignored in both of them. For an OFDM-based CR networks, several waterfilling factor aided search (WFAS) methods were derived in [11] to maximize $\mathrm{EE}$ under the minimum rate requirement constraint and average interference power constraint.

Although the above power allocation schemes are very efficient, they require to know perfect intersystem channel state information (CSI) and intrasystem CSI. The acquisition of the instantaneous intersystem CSI is difficult to obtain due to not only the lack of cooperation, but also wastes of network resources.

In this paper, we study the EE optimization problem for OFDM-based CR networks with partial intersystem CSI while ensuring a certain data rate of SU. We first analyze the relationship between $\mathrm{SE}$ and $\mathrm{EE}$ and solve this optimization problem with the help of bisection search technique. However, the accuracy of optimal solution is dependent on the number of iterations. In order to derive the exact optimal 
solution, we propose a new algorithm to maximize EE, which is different from all existing methods.

The remainder of the paper is organized as follows. Section 2 introduces an OFDM-based CR system model and provides the EE optimization problem. In Section 3, a bisection search aided energy efficient power allocation algorithm is described. To achieve the exact optimal solution, a new method with closed-form expression is developed to obtain the maximum EE. In Sections 4 and 5, simulation results and conclusions are given, respectively.

\section{System Model}

Consider an OFDM-based CR network where an SU coexists with a PU over the same spectrum band with $N$ subchannels indexed by $\mathcal{N}=\{1, \ldots, n, \ldots, N\}$. Let $h_{n}^{s s}$ be the instantaneous channel frequency response from SU-Tx to SU$\mathrm{Rx}$ on the $n$th subchannel. In this paper, we assume that $h_{n}^{s s}$ is perfectly known to the SU-Tx. However, compared with intrasystem CSI, the instantaneous channel frequency response $h_{n}^{s p}$ between SU-Tx and PU-Rx is difficult to obtain due to the lack of intersystem cooperation. We assume that only statistic distribution of intersystem CSI is available at the SU-Tx. Under an uncorrelated fading channel model [7], $h_{n}^{s s}$ and $h_{n}^{s p}$ are independent and identically distributed (i.i.d.) complex Gaussian random variables with $\mathscr{C} \mathscr{N}\left(0, \lambda_{1}\right)$ and $\mathscr{C} \mathscr{N}\left(0, \lambda_{2}\right)$, respectively.

For a spectrum sharing scenario, the PU and SU will interfere with each other. In a practical network with a sufficiently large number of subchannels, the interference introduced by PU-Tx into SU-Rx can be modeled as an additive white Gaussian noise (AWGN). This assumption is widely treated and justified in $[7,11]$. Let $p_{n}$ be the transmit power on the $n$th subchannel, and the data rate can be given by

$$
r_{n}=B \log _{2}\left(1+g_{n} p_{n}\right)
$$

where $B$ is the bandwidth of each subchannel, $g_{n}=\left|h_{n}^{s s}\right|^{2} / \sigma^{2}$ is the channel gain to noise ratio (CNR) on the $n$th subchannel and $\sigma^{2}$ denotes the variance of complex white Gaussian noise.

Consequently, the overall data rate $R$ and transmit power $P_{t}$ can be expressed as

$$
\begin{aligned}
R & =\sum_{n \in \mathcal{N}} r_{n}, \\
P_{t} & =\sum_{n \in \mathcal{N}} p_{n},
\end{aligned}
$$

respectively.

In the transmission mode of SU-Tx, the energy consumption consists of two parts: the energy consumption of power amplifier related to transmit power and the circuit energy consumption incurred by signal processing and active circuit blocks. According to [12], the circuit power, $P_{c}$, can be modeled as a linear function of data rate. We have

$$
P_{c}=P_{s}+\beta R,
$$

where $P_{s}$ is the static circuit power and $\beta$ is the dynamic circuit power per unit data rate.

In order to prevent the PU from severe performance degradation, the interference power should be restricted by

$$
E\left[\left|h_{n}^{s p}\right|^{2} p_{n}\right] \leq I, \quad \forall n \in \mathcal{N}
$$

where $I$ is the interference threshold that the PU can tolerate.

Since the random variable $X=\left|h_{n}^{s p}\right|^{2} p_{n}$ follows the chisquare distribution with mean

$$
E\left[\left|h_{n}^{s p}\right|^{2} p_{n}\right]=p_{n} \lambda_{2}
$$

then the equivalent constraint of (5) can be expressed as

$$
p_{n} \leq p_{o}=\frac{I}{\lambda_{2}}
$$

To maximize the EE of the CR network while guaranteeing that the interference to the $\mathrm{PU}$ receiver is maintained below a predefined threshold, we formulate a transmit power allocation optimization problem under some practical constraints. The optimization problem is formulated as follows:

$$
\text { P1: } \begin{aligned}
\max _{p_{n}, n \in \mathcal{N}} U & =\frac{R}{\alpha P_{t}+P_{c}}, \\
\text { s.t. } \quad R & \geq \check{R}, \\
P_{t} & \leq \widehat{P}, \\
p_{n} & \leq p_{o}, \quad \forall n \in \mathcal{N},
\end{aligned}
$$

where $\alpha$ (e.g., $\alpha>1$ ) is the reciprocal of drain efficiency of power amplifier, $\check{R}$ is the minimum rate requirement, and $\widehat{P}$ is the maximum allowable transmit power. Equation $(8 \mathrm{~b})$ is the minimum rate requirement constraint to ensure a certain QoS of SU. Equation (8c) is the interference constraint to prevent CR network from causing severe interference to PU. Equation (8d) is the total transmit power constraint at SU-TX.

It is possible that problem P1 does not have any feasible solution if $\check{R}$ cannot be achieved subject to the constraints (8c) and (8d). In this case, the SU-Tx may have to decrease $\check{R}$ to make the solution feasible. For simplicity, we assume that the minimum rate requirement $\check{R}$ is achievable under the constraints (8c) and (8d).

\section{Energy Efficient Power Allocation}

After formulating the EE optimization problem, we investigate the relation between $\mathrm{EE}$ and $\mathrm{SE}$ and then propose two optimal power allocation schemes to maximize EE.

First we define a data rate-adaptive (RA) optimization problem for the OFDM-based CR network as

$$
\begin{array}{ll}
\text { P2: } \quad \max _{p_{n}, n \in \mathcal{N}} R, \\
\text { s.t. } \quad P_{t} \leq \widehat{P}, \\
& p_{n} \leq p_{o}, \quad \forall n \in \mathcal{N} .
\end{array}
$$


It can be seen that the objectives in (8a)-(8d) and (9a)(9c) conflict with each other sometimes. Without loss of generality, assume that $g_{1} \geq g_{2} \geq \cdots \geq g_{N}$. According to [4], the optimal power allocation is achieved through waterfilling method; that is,

$$
p_{n}=\left[\mu-\frac{1}{g_{n}}\right]_{0}^{p_{o}},
$$

for all $n \in \mathcal{N}$, where $[x]_{a}^{b}=\min (b, \max (x, a))$ and $\mu$ is the water level.

Since $R$ is a strictly increasing function of $\mu$ in the feasible region, it is easy to see that $R$ has a unique value for all $P_{t} \in$ $\left[0, N p_{o}\right]$.

Lemma 1. For problem P2 without peak interference power constraint $(9 \mathrm{c})$, the optimal water level $\mu$ to maximize data rate is

$$
\mu=\min _{1 \leq m \leq N} \frac{1}{m}\left(P_{t}+\sum_{n=1}^{m} \frac{1}{g_{n}}\right) .
$$

Proof. Please see Appendix B in [13].

Based on Lemma 1, the optimal water level $\mu_{\max }$ corresponding to problem $\mathrm{P} 2$ can be obtained with iterative water filling.

If the overall data rate is fixed, the objective of problem P1 is equivalent to a transmit power-adaptive (PA) optimization problem. The optimization problem can be formulated as follows:

$$
\begin{array}{ll}
\text { P3: } & \min _{p_{n}, n \in \mathcal{N}} P_{t}, \\
\text { s.t. } \quad & R \geq \check{R}, \\
& p_{n} \leq p_{o}, \quad \forall n \in \mathscr{N} .
\end{array}
$$

Lemma 2. For problem P3 without peak interference power constraint (12c), the optimal water level $\mu$ to minimize transmit power rate is

$$
\mu=\min _{1 \leq m \leq N} 2^{\check{R} / m B}\left(\prod_{n=1}^{m} \frac{1}{g_{n}}\right)^{1 / m} .
$$

Proof. Please see Appendix A in [13].

According to Lemma 2, we can derive the optimal water level $\mu_{\min }$ to minimize $P_{t}$ using the similar way to problem P2.

\subsection{Bisection Search Aided Algorithm (BSAA)}

Lemma 3. For problem $P 1$ without constraints (8b)-(8d), the $E E, U$, is a quasiconcave function of water level $\mu$.

Proof. Please see Appendix A.

Since EE is a quasiconcave function of $\mu$ without considering $(8 b)-(8 d)$, there exists a globally optimal water level
Step 1. Initialization, $\mu_{\mathrm{opt}} \leftarrow 0$.

Step 2. Calculate the optimal water level $\mu_{\min }$ and $\mu_{\max }$,

and set $\mu_{\text {low }} \leftarrow \mu_{\text {min }}$ and $\mu_{\text {high }} \leftarrow \mu_{\text {max }}$.

Step 3. Set $\mu_{\text {mid }} \leftarrow(1 / 2)\left(\mu_{\text {low }}+\mu_{\text {high }}\right)$.

Step 4. If its derivative $d U /\left.d \mu\right|_{\mu=\mu_{\text {mid }}}>0$, then

$\mu_{\text {low }} \leftarrow \mu_{\text {mid }}$, else $\mu_{\text {high }} \leftarrow \mu_{\text {mid }}$.

Step 5. Repeat Step 4 until $\mu_{\text {high }}-\mu_{\text {low }} \leq \xi$ where $\xi$

(e.g. $\xi=0.001$ ) is a small positive constant to control the convergence accuracy.

Step 6. Finish, the optimal water level solution is

$\mu_{\text {opt }} \leftarrow \mu_{\text {mid }}$.

Algorithm 1: Bisection search aided algorithm (BSAA) for Problem P4.

$\mu_{\text {un }}$ to maximize EE. More specifically speaking, $U$ is a monotonically increasing function of $\mu$ when $\mu<\mu_{\mathrm{un}}$ and is a strictly decreasing function of $\mu$ when $\mu>\mu_{\mathrm{un}}$.

Based on the above discussion, both $\mu_{\min }$ and $\mu_{\max }$ can be acted as the lower bound and upper bound of feasible region for problem $\mathrm{P} 1$, respectively. Hence, the equivalent problem of $\mathrm{P} 1$ can be given as

$$
\begin{array}{ll}
\text { P4: } \quad \max _{\mu} U(\mu)=\frac{R}{\alpha P_{t}+P_{c}}, \\
\text { s.t. } \quad \mu_{\min } \leq \mu \leq \mu_{\max }, \\
\\
p_{n} \leq p_{o}, \quad \forall n \in \mathcal{N} .
\end{array}
$$

The optimal water level $\mu_{\text {opt }}$ can be solved by line-search techniques, for example, bisection search, which requires a predictable number of iterations and is suitable for practical implementation. Here we list the bisection search aided algorithm (BSAA) in Algorithm 1.

The key work of Algorithm 1 is to determine the sign of $\mathrm{d} U / \mathrm{d} \mu$. It can be calculated by $U(\mu+\Delta \mu)-U(\mu)$ where $\Delta \mu$ is an infinitely small positive constant. The number of iterations to determine the optimal water level $\mu_{\text {opt }}$ is $\left\lceil\log _{2}\left(\left(\mu_{\max }-\right.\right.\right.$ $\left.\left.\left.\mu_{\min }\right) / \xi\right)\right\rceil$, where $\lceil x\rceil$ denotes the smallest integer not less than $x$.

3.2. Exact Power Allocation Algorithm (EPAA). Although the optimization problem $\mathrm{P} 4$ can be easily solved by the search algorithm, the accuracy of solution is primarily dependent on $\xi$. In this subsection, a new energy efficient power allocation is proposed to achieve the exact optimal solution.

Lemma 4. For problem P4 without constraints (14b) and (14c), the optimal water level $\mu_{u n}$ to maximize EE is

$$
\mu_{u n}=\frac{e}{G} \exp \left[W_{0}\left(\frac{P_{s} G}{\alpha \widetilde{N} e}-\frac{g G}{e}\right)\right]
$$

where $e$ is the base of the natural logarithm, $W_{0}(\cdot)$ denotes the real branch of the Lambert function [14], and $\widetilde{N}$ is the number 
Step 1. Initialization, $\tilde{\mu} \leftarrow 0, \mathscr{A} \leftarrow\left\{g_{1}, g_{2}, \ldots, g_{N}\right\}$,

$R \leftarrow 0, P_{t} \leftarrow 0$.

Step 2. Using the similar way to (15), we can obtain the optimal unconstrained water level as

$\mu_{\mathrm{un}} \leftarrow \frac{2^{-R / m B} e}{G} \exp \left[W_{0}\left(\left(\frac{\alpha P_{t}+P_{s}}{\alpha m}-g\right) \frac{G}{e} 2^{R / m B}\right)\right]$, where

$G \leftarrow\left(\prod_{n=1}^{m} \mathscr{A}_{n}\right)^{1 / m}, g \leftarrow \frac{1}{m} \sum_{n=1}^{m} \frac{1}{\mathscr{A}_{n}}, \mathcal{S} \leftarrow\left\{n \mid \mu_{\mathrm{un}}>\frac{1}{\mathscr{A}_{n}}\right\}$

and $m \leftarrow|\mathcal{S}|$.

Step 3. Select, $\widehat{n} \leftarrow \arg \max _{n}\left\{\mathscr{A}_{n}\right\}$.

Step 4. Remove and update, if $\tilde{\mu}-1 / \mathscr{A}_{\hat{n}}>p_{o}$, then

$\mathscr{A} \leftarrow \mathscr{A} \backslash\left\{\mathscr{A}_{\hat{n}}\right\}, R \leftarrow R+B \log _{2}\left(1+p_{o} \mathscr{A}_{\hat{n}}\right)$,

$P_{t} \leftarrow P_{t}+p_{o}$ and goto Step 2; else goto Step 5 .

Step 5. Optimal power allocation for each subchannel,

$p_{n} \leftarrow\left[\mu_{\mathrm{opt}}-\frac{1}{g_{n}}\right]_{0}^{p_{o}}$, where

$\mu_{\text {opt }} \leftarrow \min \left(\mu_{\max }, \max \left(\mu_{\mathrm{un}}, \mu_{\min }\right)\right)$.

Algorithm 2: Exact power allocation algorithm (EPAA) for Problem P4.

of subcarriers with transmit power $p_{n}>0 . g$ and $G$ are defined as

$$
\begin{gathered}
g=\frac{1}{\widetilde{N}} \sum_{n=1}^{\widetilde{N}} \frac{1}{\mathscr{A}_{n},} \\
G=\left(\prod_{n=1}^{\widetilde{N}} \mathscr{A}_{n}\right)^{1 / \widetilde{N}},
\end{gathered}
$$

respectively, where $\mathscr{A}=\left\{g_{1}, g_{2}, \ldots, g_{N}\right\}$.

Proof. Please see Appendix B.

However, the optimal unconstrained water level $\mu_{\text {un }}$ may lead to the transmit power of some subchannels exceeding peak power $p_{o}$. To solve this problem, we should pour transmit power to these subcarriers in prior and keep the allocated power of all subchannels satisfying $p_{n} \leq p_{o}, \forall n \in$ $\mathcal{N}$. The pseudocode of this power allocation algorithm is presented in Algorithm 2.

\section{Simulation Results}

Simulation results are provided to corroborate our analysis and to demonstrate the performance of the proposed scheme. We evaluate the EE and SE performance of proposed schemes and compare them with conventional RA and PA power allocation schemes. The minimum SE requirement is $0.5 \mathrm{bits} / \mathrm{s} / \mathrm{Hz}$. The other simulation parameters are set as follows: $B=15 \mathrm{kHz}, N=128, \lambda=\lambda_{1} / \lambda_{2}$ (we fix $\lambda_{2}=1$ and vary $\left.\lambda_{1}\right), \widehat{P}=3 \mathrm{~W}, \sigma^{2}=0.01 \mathrm{~W}, I=\rho \sigma^{2}, P_{s}=0.8 \mathrm{~W}$, $\alpha=2$, and $\beta=0.2 \mathrm{~W} / \mathrm{Mbps}$.

Figure 1 shows the EE performance of different schemes. In this simulation example, the interference tolerance is $I=\sigma^{2}$. Compared with conventional RA and PA power allocation schemes, the proposed schemes achieve the best EE performance. For different power allocation schemes, the EE

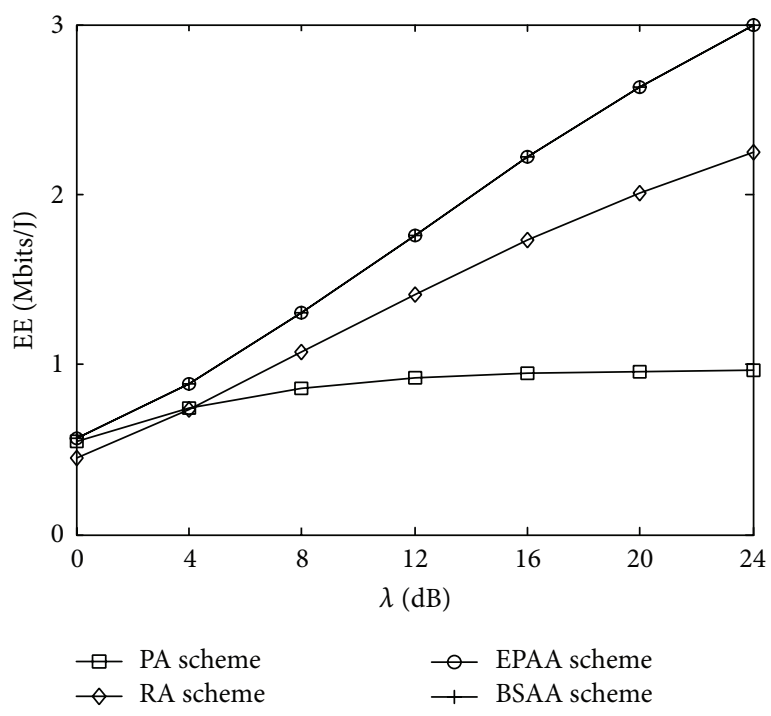

FIGURE 1: EE-versus-channel gain ratio $\lambda, \rho=1$.

increases with the relative channel gain ratio $\lambda$. Figure 2 plots the SE-versus- $\lambda$ curves corresponding to Figure 1 . It can be seen that the EE continuously increases as $\lambda$ increases, which means as the CNR becomes larger, less transmit power has to be consumed to achieve the same data rate, and as a result, the EE increases. We can see that the proposed schemes can achieve higher EE with less power consumption at the cost of some SE loss when compared with the RA scheme.

Figures 3 and 4 show the impact of interference tolerance on the power allocation results where the relative channel gain ratio is $\lambda=10 \mathrm{~dB}$. It can be seen that all schemes may have to operate exactly at the maximum allowable transmit power to meet the minimum rate requirement when $\rho$ is small. It indicates that for the different interference tolerance constraints, which are larger than or equal to a certain 


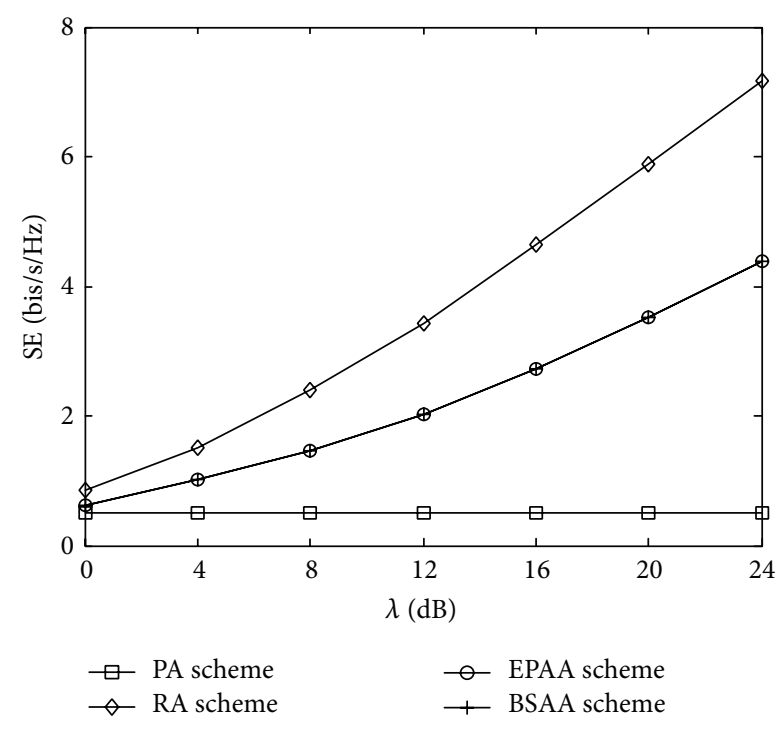

FIGURE 2: SE-versus-channel gain ratio $\lambda, \rho=1$.

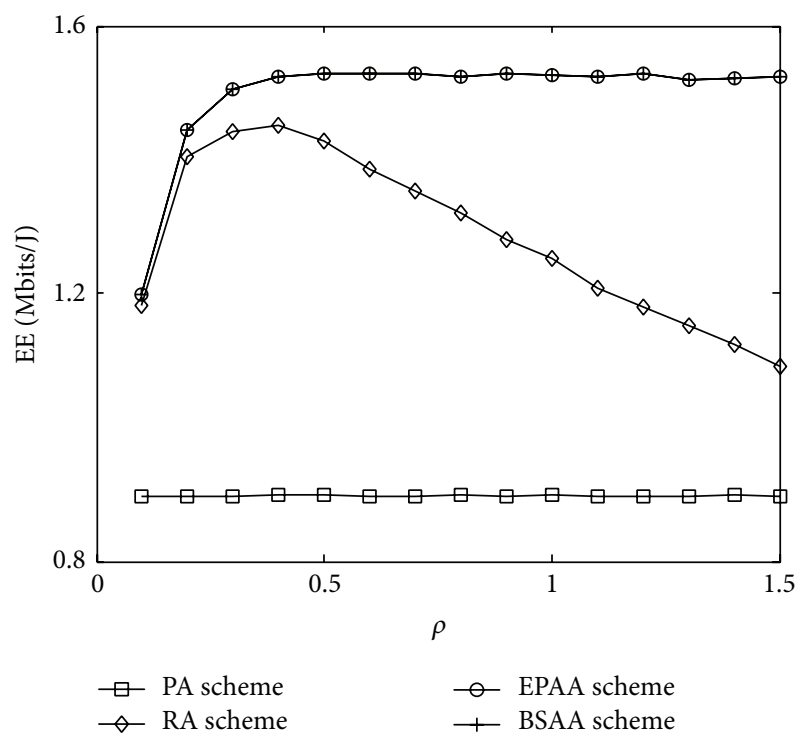

FIGURE 3: EE-versus-interference tolerance $\rho, \lambda=10 \mathrm{~dB}$.

threshold, both BSAA and EPAA schemes lead to a same SE. However, for the interference tolerance that is smaller than the threshold, both the EE and SE of the proposed schemes increase with $\rho$. Once the highest $\mathrm{EE}$ is achieved, the data rate as well as the transmit power consumption of the proposed schemes remains the same and no more transmit power will be used even when the interference tolerance continues to increase. In contrast to optimal power allocation schemes, the SE of RA scheme greedily grows with the interference tolerance all the time until the total transmit power is used up.

In Figures 5 and 6, we demonstrate the impact of the minimum SE requirement, $\eta_{\min }=\breve{R} / N B$, on the system performances in the case that $\lambda=10 \mathrm{~dB}$ and $\rho=2$. We can divide the minimum SE region into two parts based on

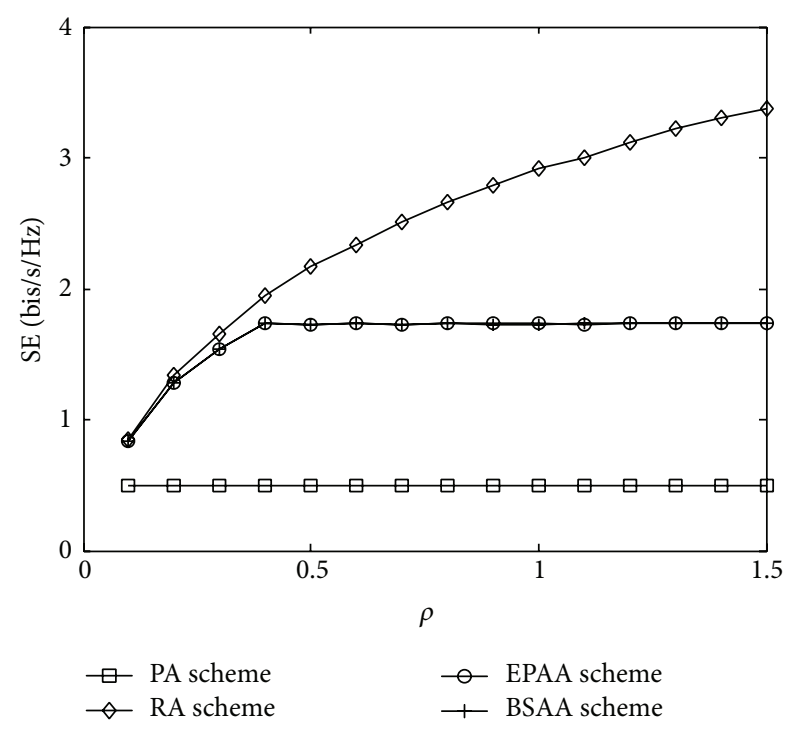

FIgURE 4: SE-versus-interference tolerance $\rho, \lambda=10 \mathrm{~dB}$.

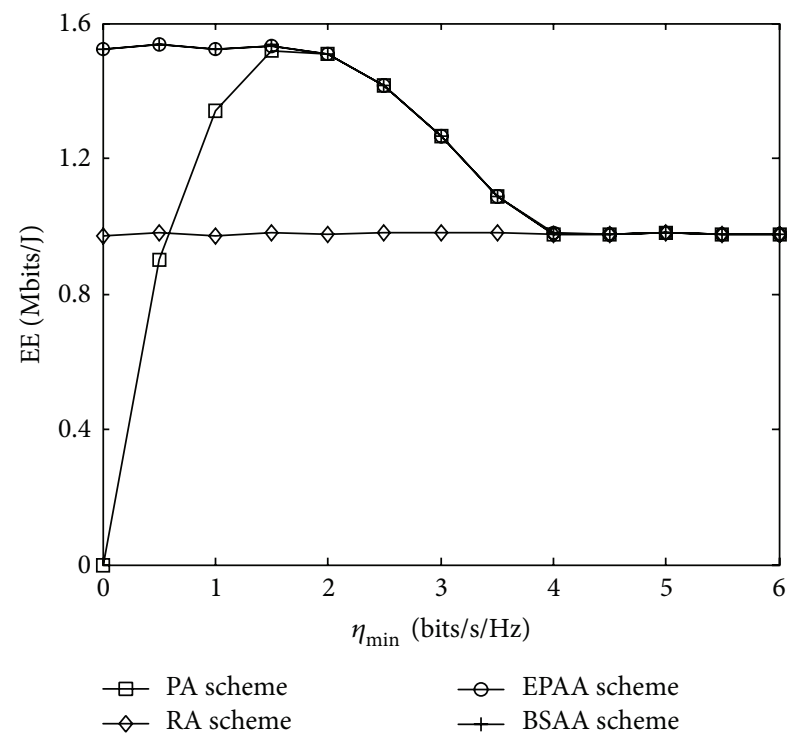

FIGURE 5: EE-versus-the minimum SE requirement $\eta_{\min }, \lambda=10 \mathrm{~dB}$, $\rho=2$.

whether $(8 b)$ is active. It can be observed that for the different minimum throughput requirements, $\eta_{\min }$, which are smaller than or equal to a certain threshold (i.e., $1.8 \mathrm{bits} / \mathrm{s} / \mathrm{Hz}$ ), energy efficient designs lead to the same system performance. However, for the minimum SE requirement that is larger than the threshold, the proposed schemes are overlapped with the PA scheme. Furthermore, we can see that the optimal EE decreases with $\eta_{\min }$ while SE increases in the case that $1.8 \mathrm{bits} / \mathrm{s} / \mathrm{Hz} \leq \eta_{\min } \leq 4.0 \mathrm{bits} / \mathrm{s} / \mathrm{Hz}$. This is because the optimal energy efficient designs are to operate exactly at the required minimum data rate requirement. Hence, there exists a tradeoff between the EE and SE in the active region of $\eta_{\min }$. Note that once the highest achievable SE is obtained, the $\mathrm{EE}$ as well as the power consumption remains the same 


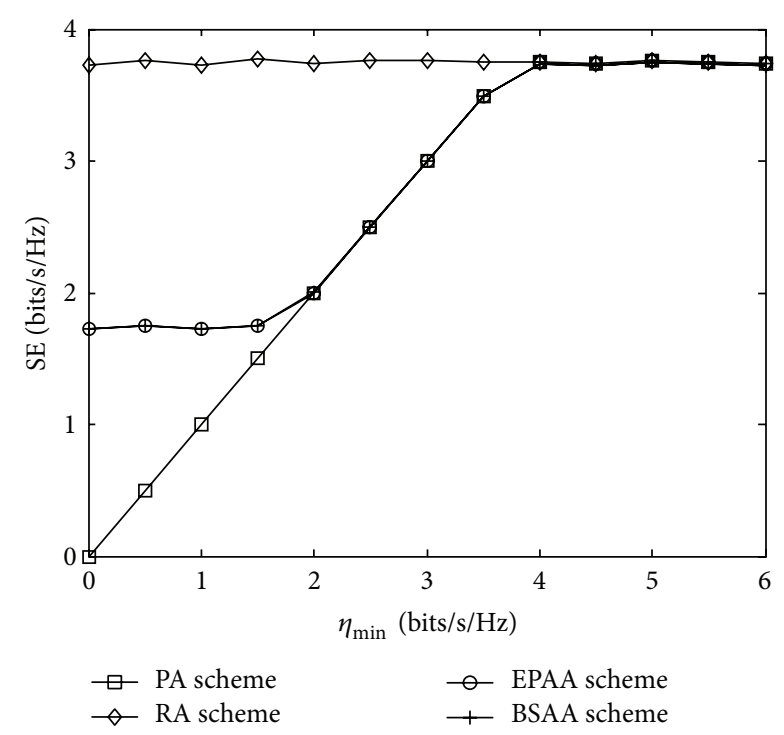

FIGURE 6: SE-versus-the minimum SE requirement $\eta_{\min }, \lambda=10 \mathrm{~dB}$, $\rho=2$.

and no more power will be used even when the minimum SE requirement $\eta_{\min }$ continues to increase for the energy efficient designs.

\section{Conclusion}

We consider the energy efficient power allocation problem of OFDM-based CR systems with statistical intersystem CSI available. We first introduce two conventional optimization problems: rate-adaptive (RA) problem and power-adaptive (PA) problem. After analyzing the relationship between EE and SE, an efficient power allocation scheme with the help of bisection search method has been proposed to maximize EE. However, the accuracy of the solution is dependent on the number of iterations. To derive the exact optimal performance, we also propose a new power allocation algorithm which is different from all existing methods. Simulation results confirm considerable EE improvement by the proposed schemes.

In future, the energy efficient resource allocation problems for more complicated CR networks (e.g., multiuser scenario with imperfect channel state information) should be considered. We may jointly extend the proposed schemes and the methods in [15] to address these optimization problems.

\section{Appendix}

\section{A. Proof of Lemma 1}

From problem $\mathrm{P} 1$, the objective of EE optimization is modeled as

$$
\max _{p_{n}} U\left(P_{t}\right)=\frac{1}{\left(\left(\alpha P_{t}+P_{s}\right) / R\left(P_{t}\right)\right)+\beta} .
$$

Since $\alpha>0, R\left(P_{t}\right)>0, P_{t}>0$, and $P_{s}>0$, the EE $U$ is a strictly monotonic decreasing function of $\left(\alpha P_{t}+P_{s}\right) / R$. Then the equivalent objective can be reformulated as

$$
\max _{p_{n}} \bar{U}\left(P_{t}\right)=\frac{R\left(P_{t}\right)}{\alpha P_{t}+P_{s}} .
$$

For arbitrary $\theta \in(0,1)$, since $R\left(P_{t}\right)$ is concave function of $P_{t}$, we have

$$
\begin{aligned}
\bar{U}\left(\theta x_{1}+(1-\theta) x_{2}\right) & \geq \frac{\theta R\left(x_{1}\right)+(1-\theta) R\left(x_{2}\right)}{\theta\left(\alpha x_{1}+P_{s}\right)+(1-\theta)\left(\alpha x_{2}+P_{s}\right)} \\
& \geq \min \left(\bar{U}\left(x_{1}\right), \bar{U}\left(x_{2}\right)\right) .
\end{aligned}
$$

According to [13], we can conclude that $U$ is quasiconcave for $P_{t} \geq 0$. Since $P_{t}$ is a monotonic increasing function of water level without (7), hence, EE is a quasiconcave function of water level.

\section{B. Proof of Lemma 4}

According to Lemma 3, energy efficiency is a quasiconcave function of water level. Hence, a unique unconstrained optimal water level corresponding to P4 without (14b) and (14c) always exists. The true water level is assumed to be $\mu$ and the transmit power on the $n$th subcarrier is

$$
p_{n}= \begin{cases}\mu-\frac{1}{g_{n}}, & 1 \leq n \leq \widetilde{N} \\ 0, & \widetilde{N}<n \leq N,\end{cases}
$$

where $\widetilde{N}$ is the number of subcarriers with transmit power $p_{n}>0$.

Denote that $\mathscr{A}=\left\{g_{1}, g_{2}, \ldots, g_{N}\right\}$ is the CNR set of all subcarriers. Substituting (B.1) into (2) and (3), we can obtain the overall data rate $R$ and total transmit power $P_{t}$ as

$$
\begin{aligned}
R & =B \sum_{1 \leq n \leq \widetilde{N}} \log _{2}\left(\mu g_{n}\right) \\
& =\widetilde{N} B \log _{2}(\mu G), \\
P_{t} & =\widetilde{N} \mu-\sum_{1 \leq n \leq \widetilde{N}} \frac{1}{g_{n}} \\
& =\widetilde{N}(\mu-g),
\end{aligned}
$$

respectively, where

$$
\begin{gathered}
G=\left(\prod_{n=1}^{\widetilde{N}} \mathscr{A}_{n}\right)^{1 / \widetilde{N}}, \\
g=\frac{1}{\widetilde{N}} \sum_{n=1}^{\widetilde{N}} \frac{1}{\mathscr{A}_{n}} .
\end{gathered}
$$


Thus the objective function, (14a), can be given as

$$
\begin{aligned}
U & =\frac{R}{\alpha P_{t}+P_{c}} \\
& =\frac{\widetilde{N} B \log _{2}(\mu G)}{\alpha \widetilde{N}(\mu-g)+P_{s}+\widetilde{N} B \log _{2}(\mu G)} .
\end{aligned}
$$

Differentiating the objective function, (B.5), with respect to $\tilde{\mu}$, we have

$$
\frac{\partial U}{\partial \mu}=F\left(1+\frac{P_{s}}{\alpha \widetilde{N} \mu}-\frac{g}{\mu}-\log _{e}(\mu G)\right),
$$

where $e$ is the base of the natural logarithm and

$$
F=\frac{B}{\alpha \log _{e} 2\left(\mu-g+P_{s} / \alpha \widetilde{N}+\beta B \log _{e}(\mu G) / \alpha \log _{e} 2\right)^{2}}
$$

It is obvious that $F$ is a positive variable. Setting the derivative, (B.6), to zero, we can obtain

$$
\frac{P_{s} G}{\alpha \widetilde{N} e}-\frac{g G}{e}=\frac{\mu G}{e} \log _{e}\left(\frac{\mu G}{e}\right) .
$$

Let $X=\log _{e}(\mu G / e)$ and $Y=P_{s} G / \alpha \widetilde{N} e-g G / e$; then (B.8) can be expressed as

$$
X e^{X}=Y .
$$

Its solution is $X=W_{0}(Y)$, where $W_{0}(\cdot)$ denotes the real branch of the Lambert function [14]. Thus, the optimal unconstrained water level $\tilde{\mu}$ is

$$
\tilde{\mu}=\frac{e}{G} \exp \left[W_{0}\left(\frac{P_{s} G}{\alpha \widetilde{N} e}-\frac{g G}{e}\right)\right] .
$$

Here Lemma 4 is proved.

\section{Conflict of Interests}

The authors declare that there is no conflict of interests regarding the publication of this paper.

\section{Acknowledgment}

The work in this paper has been supported by funding from Beijing Natural Science Foundation under Grant no. 4122009.

\section{References}

[1] D. Feng, C. Jiang, G. Lim, L. J. Cimini, G. Feng, and G. Y. Li, "A survey of energy-efficient wireless communications," IEEE Communications Surveys \& Tutorials, vol. 15, no. 1, pp. 167-178, 2013.

[2] S. Haykin, "Cognitive radio: brain-empowered wireless communications," IEEE Journal on Selected Areas in Communications, vol. 23, no. 2, pp. 201-220, 2005.
[3] T. A. Weiss and F. K. Jondral, "Spectrum pooling: an innovative strategy for the enhancement of spectrum efficiency," IEEE Communications Magazine, vol. 42, no. 3, pp. S8-S14, 2004.

[4] R. Zhang, Y.-C. Liang, and S. Cui, "Dynamic resource allocation in cognitive radio networks," IEEE Signal Processing Magazine, vol. 27, no. 3, pp. 102-114, 2010.

[5] P. Wang, M. Zhao, L. Xiao, S. Zhou, and J. Wang, "Power allocation in OFDM-based cognitive radio systems," in Proceedings of the 50th Annual IEEE Global Telecommunications Conference (GLOBECOM '07), pp. 4061-4065, November 2007.

[6] G. Bansal, M. J. Hossain, and V. K. Bhargava, "Optimal and suboptimal power allocation schemes for OFDM-based cognitive radio systems," IEEE Transactions on Wireless Communications, vol. 7, no. 11, pp. 4710-4718, 2008.

[7] K. Son, B. C. Jung, S. Chong, and D. K. Sung, "Power allocation for OFDM-based cognitive radio systems under outage constraints," in Proceedings of the IEEE International Conference on Communications (ICC '10), May 2010.

[8] C. Xiong, G. Y. Li, S. Zhang, Y. Chen, and S. Xu, "Energyefficient resource allocation in OFDMA networks," IEEE Transactions on Communications, vol. 60, no. 12, pp. 3767-3778, 2012.

[9] Y. Li, X. Zhou, H. Xu, G. Y. Li, D. Wang, and A. Soong, "Energy-efficient transmission in cognitive radio networks," in Proceedings of the 7th IEEE Consumer Communications and Networking Conference (CCNC '10), January 2010.

[10] R. Xie, F. R. Yu, H. Ji, and Y. Li, "Energy-efficient resource allocation for hrterogeneous cognitive radio networks with femtocells," IEEE Transactions on Wireless Communications, vol. 11, no. 11, pp. 3910-3920, 2012.

[11] J. Mao, G. Xie, J. Gao, and Y. Liu, "Energy efficiency optimization for OFDM-based cognitive radio systems: a water-filling factor aided search method," IEEE Transactions on Wireless Communications, vol. 12, no. 5, pp. 2366-2375, 2013.

[12] G. Miao, N. Himayat, and G. Y. Li, "Energy-efficient link adaptation in frequency-selective channels," IEEE Transactions on Communications, vol. 58, no. 2, pp. 545-554, 2010.

[13] Y. Li, Z. Zheng, M. Zhao, S. Jin, and Z. Huang, "Chunkbased energy efficient resource allocation in OFDMA systems," Mathematical Problems in Engineering, vol. 2013, Article ID 613470, 7 pages, 2013.

[14] S. Boyd and L. Vandenberghe, Convex Optimization, Cambridge University Press, Cambridge, UK, 2004.

[15] G. Ding, Q. Wu, and J. Wang, "Sensing confidence levelbased joint spectrum and power allocation in cognitive radio networks," Wireless Personal Communications, vol. 72, no. 1, pp. 283-298, 2013. 


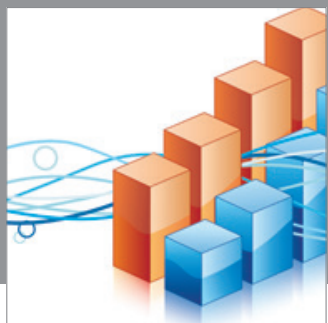

Advances in

Operations Research

mansans

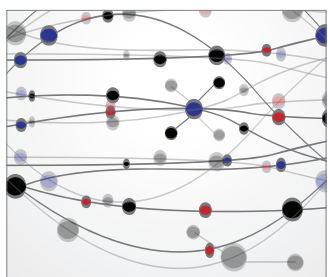

The Scientific World Journal
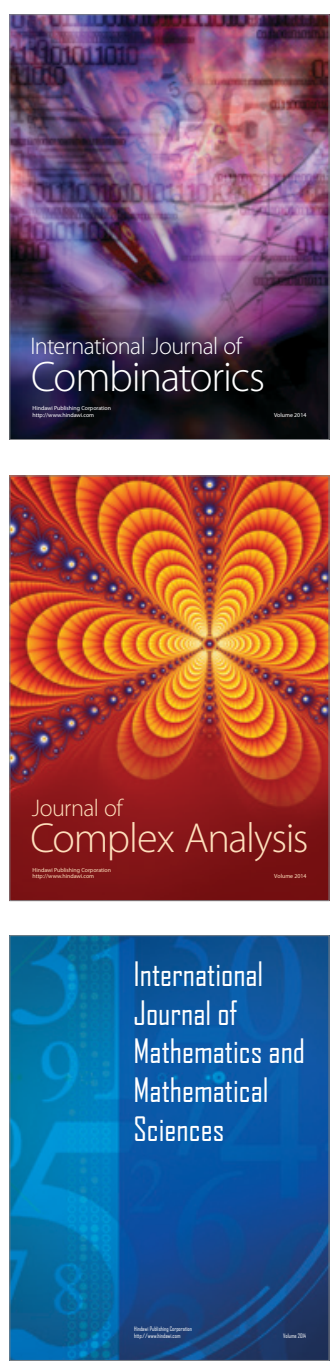
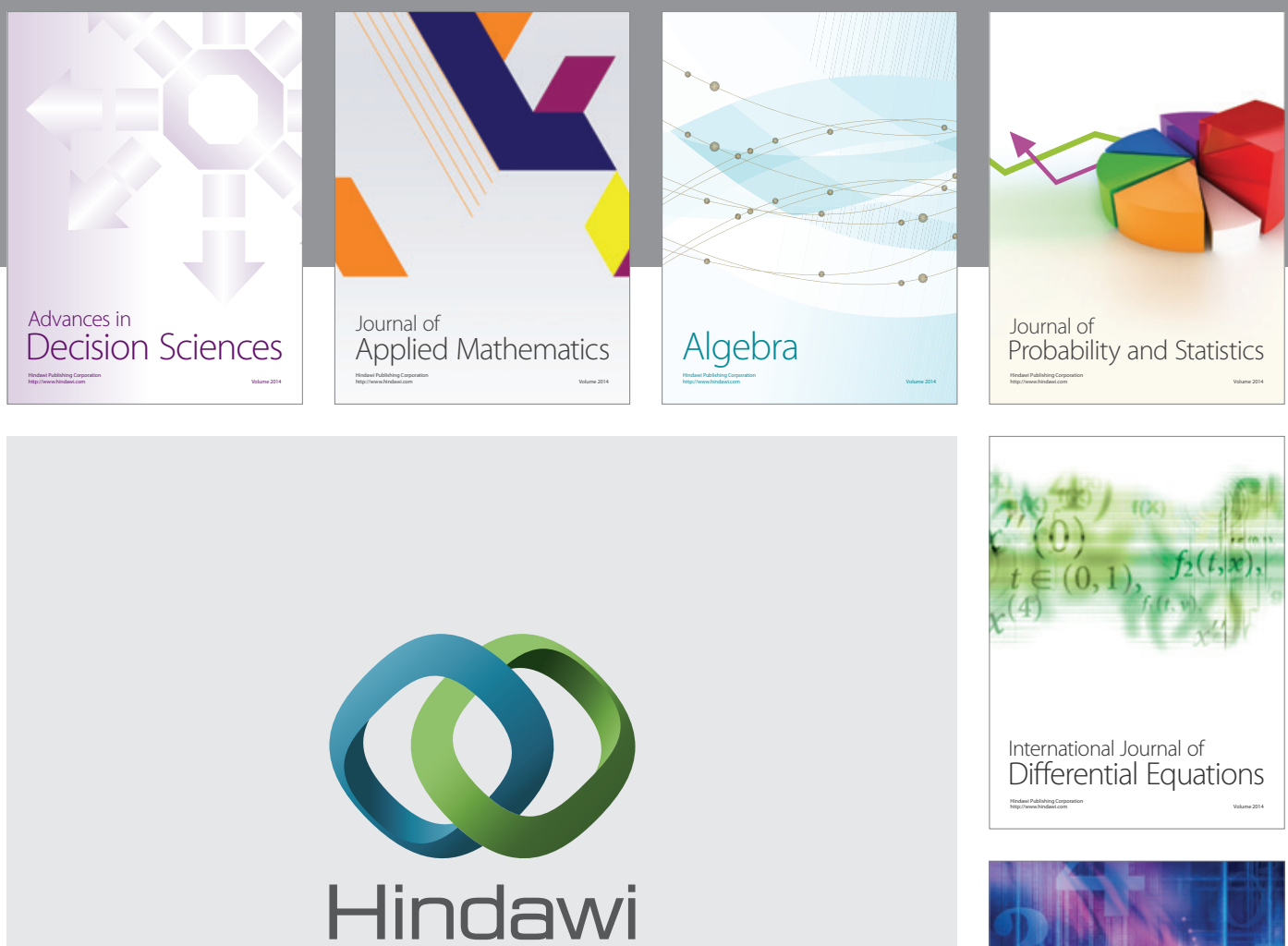

Submit your manuscripts at http://www.hindawi.com
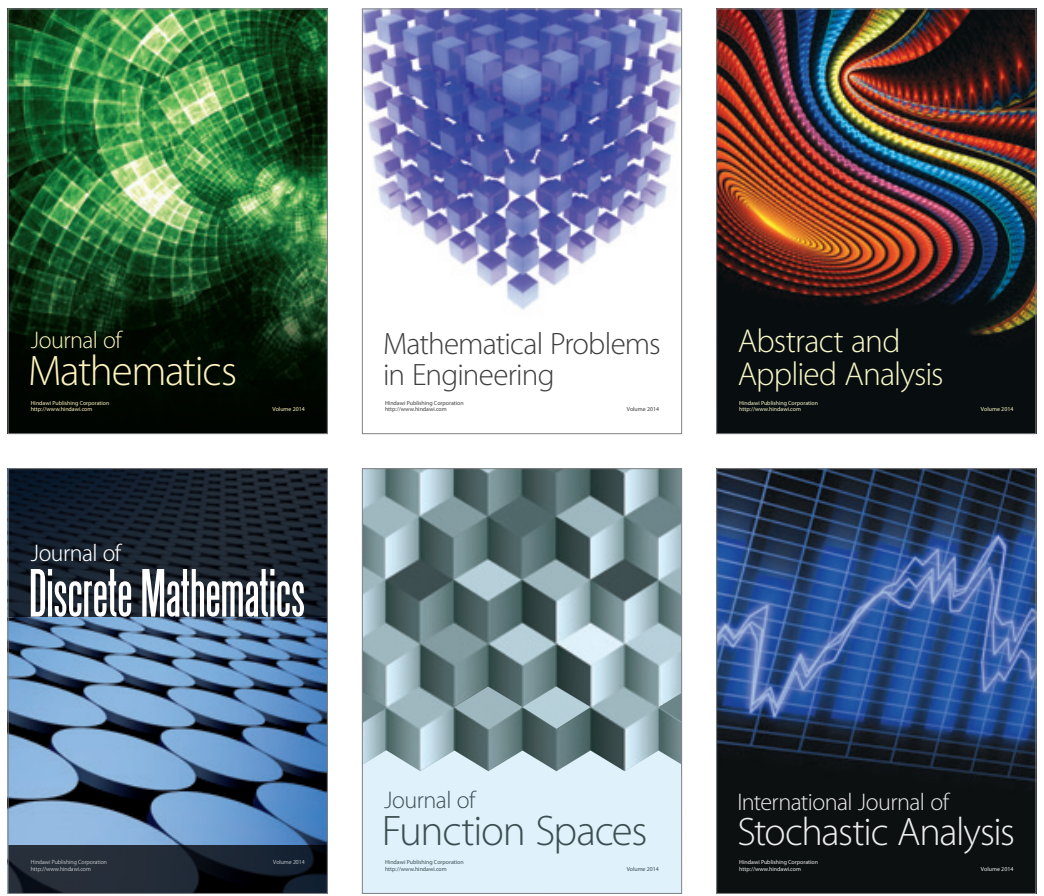

Journal of

Function Spaces

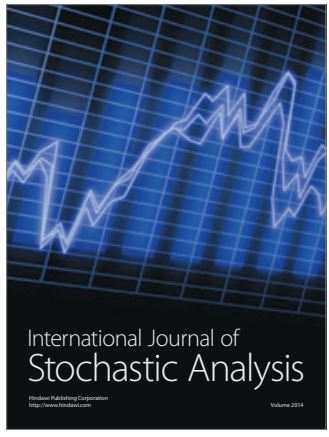

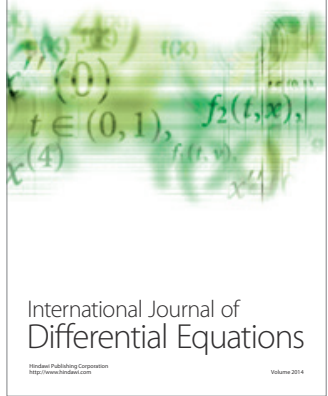
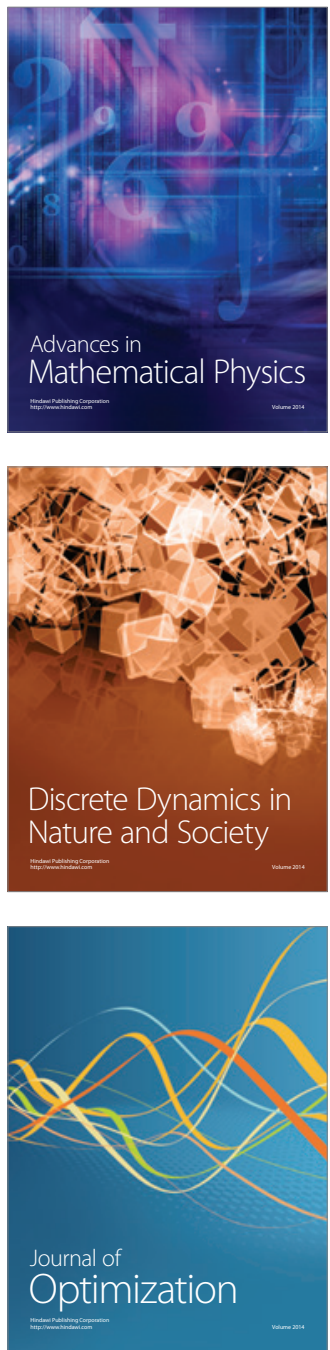